\title{
Matrices de transición y patrones de variabilidad cognitiva*
}

\section{Transition Matrix and Patterns of Cognitive Variability}

Recibido: junio 9 de 2011 | Revisado: marzo 20 de 2012 | Aceptado: junio 20 de 2012

\author{
Julio CÉSAR OSSA ** \\ Universidad de San Buenaventura, Cali, Colombia
}

doi:10.11144/Javeriana.UPSY12-2.mtpv

Para citar este artículo: Ossa, J. C. (2013). Matrices de transición y patrones de variabilidad cognitiva. Universitas Psychologica, 12(2), 559-570.

* Agradecimientos: El autor agradece a Rebeca Puche-Navarro, directora de Tesis Doctoral, y a COLCIENCIAS por el crédito condonable para adelantar estudios de posgrado en Colombia.

** Doctor en Psicología y profesor Titular en la Facultad de Psicología de la Universidad de San Buenaventura, Cali (Colombia). Integrante del Grupo de Investigación en Evaluación y Calidad de la Educación GIECE.E-mail: juceossa@gmail.com

\section{RES U MEN}

En este trabajo se aborda la variabilidad a través de la clasificación y las coordinaciones inferenciales. Participaron 34 niños de cuatro años de edad que asisten a seis jardines infantiles de la ciudad de Cali (Colombia). Se utilizó una tarea que implica el uso de hasta cinco criterios de clasificación. Se empleó el método microgenético para obtener datos detallados sobre aspectos cualitativos y cuantitativos de cambio. Para cuantificar las variaciones de los desempeños, se recurrió a las matrices de transición (derivadas de los modelos de Markov). Los resultados arrojan tres tipos de variabilidad (patrones) que responden a diferentes usos de la clasificación y diferentes niveles de coordinación inferencial. Se concluye que la variabilidad es la evidencia de los desequilibrios cognitivos.

Palabras clave autores

Clasificación, coordinaciones inferenciales, patrones de variabilidad cognitiva, matrices de transición, modelos de Markov.

Palabras clave descriptores

Psicología cognitiva, variabilidad cognitiva, patrones de variabilidad.

\section{A B S T R A C T}

This paper emphasizes the study of cognitive variability across classification and inferential coordinations. Thirty-four children (4-years-old) participated in this study who attends six kindergartens in the city of Cali-Colombia. We used a task involving the use of up to five classification criteria. A microgenetic method was used to obtain the detailed data on the qualitative and quantitative aspects of change. To quantify changes in performance we used transition matrix (derived from Markov models). The results show three types of variability (patterns) which respond to different classification criteria and the use of different levels of inferential coordination. We conclude that the variability is the evidence of cognitive imbalances and those types of variability reflect different organizational dynamics.

Key words authors

Cognitive Variability Patterns, Transition Matrix, Markov Models.

Key words plus

Cognitive Psychology, Cognitive Variability, Patterns of Variability. 


\section{Introducción}

Un interés vigente de la teoría de Piaget para la investigación actual del desarrollo cognitivo se debe, en buena parte, a su pregunta sobre los cambios que permiten el acceso a pensamientos nuevos: lo que los psicólogos llamaron las transformaciones que llevan al cambio y que constituyen el desarrollo. Piaget plantea que las transformaciones no se han podido representar mediante leyes exponenciales las cuales describen de nuevo la suma gradual de las adquisiciones y no las modificaciones de los procesos formadores que podrían explicarlas (Piaget, 1979, pp. 19-20). Piaget interroga por la razón de los desequilibrios y su función en el desarrollo; para él una de las fuentes de cambio en el desarrollo debe buscarse en los desequilibrios (Piaget, 2000).

El objetivo de este trabajo es abordar los desequilibrios cognitivos desde la perspectiva de la variabilidad cognitiva. El propósito fundamental es identificar las probabilidades de transición que subyacen a diferentes tipos de trayectorias de desempeño (de naturaleza microgenética). Para este efecto, se utilizan las matrices de transición (modelos de Markov) con el objeto de capturar las probabilidades de cambio que subyacen a los funcionamientos variables. Si bien no se trata de representar el cambio a partir de una ley general, este estudio pretende identificar algunos patrones de la variabilidad.

\section{El problema de la variabilidad}

En la literatura de los últimos años la variabilidad intra-individual (en adelante variabilidad) deja de ser un problema de medidas ${ }^{1}$ y se instaura como el eje central para explicar el desarrollo (Courage \& Howe, 2002; Goswani, 2000; Yan \& Fischer, 2002, 2007). Es por esto que se reconoce que las variaciones en el desarrollo y en el funcionamiento no son exclusivamente el resultado de error de la medida o ruido del azar (Yan \& Fischer, 2002, p. 143). La variabilidad deja de ser un ruido ajeno a la natu-

1 Problema conocido como el error de varianza. raleza de los datos para considerarse como una propiedad indisociable de los procesos de cambio en el desarrollo.

La variabilidad ha jugado un papel que ha oscilado entre la incomodidad y la promesa de ser la clave para entender el desarrollo (Miller, 2002; Puche-Navarro, 2003, 2008; Siegler, 1995, 2002a; Siegler \& Svetina, 2002, 2006; van Dijk \& van Geert, 2007; Yan \& Fischer, 2002). La relación entre la variabilidad y procesos construcción del conocimiento, se consolida "como distintos itinerarios para acceder al conocimiento" (Rose \& Fischer, 2009, p. 400).

Para Siegler (2002b) la variabilidad se presenta bajo la forma de diferentes comportamientos: frente a la misma tarea en las mismas condiciones, y en dos o más momentos cercanos en el tiempo. Las razones de la presencia de la variabilidad puede encontrarse en los propios procesos del cambio cognitivo (Siegler, 1994). La aparición de nuevas estrategias puede ser la evidencia que apunta al cambio cognitivo (Siegler, 1994, pp. 2-3) y la diversidad de estrategias indican la presencia de "mecanismos que producen cambios en el desarrollo" (Siegler, 2002b, p. 550).

La cuestión es explicar por qué el desempeño del sujeto oscila, es variable e irregular. En palabras de Miller "iPor qué un niño muestra niveles variables de desempeño cognitivo?" (Miller, 2002, p. 161). Yan y Fischer (2002) hacen énfasis en la variabilidad como característica propia del desarrollo y la definen como una fluctuación espontánea del desempeño observado en un período de tiempo corto.

Para Rose y Fischer todo el desarrollo y el funcionamiento cognitivo se presenta "como trayectorias en un flujo de cambio constante". El desarrollo y el funcionamiento cognitivo tiene dos caras: los momentos de estabilidad y congruencia, y las fluctuaciones y los itinerarios variables (Rose \& Fischer, 2009). En este sentido, la variabilidad no se identifica exclusivamente con tipos de estrategia o tipos de respuesta ya que es la concepción misma del desarrollo lo que cambia (Miller, 2002).

Piaget (1979) afirma que el paso de una forma de equilibrio a otra forma de equilibrio se presenta a diferentes ritmos de organización. La variabilidad y los diferentes puntos de inflexión de la trayecto- 
ria del desarrollo cognitivo reflejan la dinámica no lineal del cambio (Combariza \& Puche-Navarro, 2009). Es por esta razón que las leyes exponenciales no son el mejor instrumento para representar dichas transformaciones.

\section{Definición operacional de la variabilidad}

Se debe hacer énfasis en la medición y en el método utilizado para investigar el cambio en el desarrollo. Desde un punto de vista metodológico la variabilidad se mide definiendo una relación entre el dominio y el rango: donde a cada objeto del dominio se le asigna, a través de una función, un objeto del rango. El espacio muestral del dominio está constituido por todos los estados que puede tomar la variable y el espacio muestral del rango se define a partir de símbolos o valores numéricos que representan cada uno de los estados de la variable (Kerlinger \& Lee, 2002).

Desde esta perspectiva se encuentra que van Geert y van Dijk (2002) definen la variabilidad como las diferencias en el nivel de una variable (de desarrollo) entre medidas repetidas. La anterior definición puede ser leída como una función $f$ del valor de $x$ en el tiempo $t$.

La relación entre la conceptualización y la operacionalización del problema permite identificar la razón por la cual el método microgenético es una herramienta estratégica para encontrar la variabilidad (Puche-Navarro, Ossa \& Guevara, 2006; Siegler, 1995). Con el método microgenético es posible monitorear las trayectorias de la variable desempeño, con el objetivo de indagar por las fuentes de cambio y explorar la naturaleza de las transiciones (Siegler, 1995). Operacionalmente, la cuestión es capturar la mayor cantidad de datos de una variable $x$ en periodos de tiempo relativamente cortos. Conceptualmente, se trata de observar el cambio de naturaleza de un comportamiento cuando el estado de equilibrio se perturba; el interés es observar cómo se produce la organización y en este sentido hay tres posibilidades: permanecer en el mismo estado de organización, fluctuar entre dos estados de organización y cambiar a un nuevo estado de organización.
Teniendo en cuenta lo antes mencionado el presente estudio aborda el problema de la variabilidad utilizando un diseño microgenético y se propone explorar la naturaleza de la transiciones, y para ello se utilizan los modelos de Markov que serán descritos a continuación.

\section{Modelos de Markov y dinámicas de transición del cambio}

Los modelos de Markov fueron creadas por Andréi Andréyevich Márkov (1856-1922). Son una herramienta para modelar y simular el cambio, lo cual permite analizar el comportamiento de procesos estocásticos que evolucionan de forma no determinista. Es decir, son modelos que permiten hacer inferencias probabilísticas a partir de una historia, a partir de la cual la probabilidad de ocurrencia de un evento depende del evento inmediatamente anterior. Pinker (2001) sin referirse explícitamente a las cadenas de Markov define su funcionamiento sobre la base de una probabilidad estocástica (los predecible y lo aleatorio): "Una inferencia probabilística es una predicción que hoy se basa en frecuencias recogidas ayer" (Pinker, 2001, p. 451).

De acuerdo con Visser, Raijmakers y Molenaar (2002, p. 185), los modelos de Markov "se han utilizado en psicología por lo menos desde la década de 1950. Ellos se han aplicado sobre todo en las áreas de aprendizaje y la memoria. En el ámbito del aprendizaje, los modelos de Markov han demostrado ser muy flexibles para describir y formalizar el desarrollo del conocimiento".

En este estudio se utilizan los modelos de Markov para identificar las probabilidades de transición de la variabilidad. Se emplean cuatro matrices de transición (una para cada aplicación de la tarea) con el propósito de observar el cambio de las probabilidades de transición a través del tiempo. Estos modelos permiten describir cuantitativamente el cambio y las variaciones en el desempeño de los niños.

Los modelos de Markov son utilizados para describir el comportamiento en términos de probabilidad de cambio a lo largo del tiempo; en este sentido, permiten seguir la trayectoria del desempeño de un niño o un grupo de niños. Como se ha mencionado, 
el foco de interés de este estudio es la variabilidad y para seguirla se identifican las probabilidades de transición entre diferentes estados de organización y regulación. En los modelos de Markov los estados son la caracterización que puede tomar el proceso en un instante de tiempo dado.

Desde la perspectiva de la variabilidad, los cambios de estado se constituyen como el núcleo fundamental del problema, y son la base para determinar la dinámica (de cambio) del sistema. En los modelos de Markov los cambios de estado a lo largo del tiempo se denominan transición y, como se recuerda, esa es una función del método microgenético y el objetivo principal del estudio de la variabilidad (Siegler, 1995).

Los cambios de estado son cuantificados a partir de frecuencias de ocurrencia y se ordenan en una matriz de transición (Apéndice 1). Técnicamente, una matriz de transición define la probabilidad de cambio de estado a partir del tiempo actual $\left(t_{1}\right)$ hasta un tiempo futuro $\left(t_{+1}\right)$. En un modelo de Markov la probabilidad para llegar a un estado futuro solo depende del estado actual (Apéndice 2, Ecuación 1). Con un procedimiento de conteo y ordenando los datos en matrices, un modelo de Markov permite calcular las probabilidades de ocurrencia de los eventos en el futuro inmediato (Apéndice 2, Ecuación 2) o en un futuro más lejano (Apéndice 2, Ecuación 3). Una matriz sintetiza los diferentes cambios posibles en la trayectoria de los desempeños de los niños: permanencias (diagonal principal), ascensos (sobre la diagonal principal) y descensos (debajo de la diagonal principal).

Una herramienta que se deriva de los modelos de Markov son los vectores de probabilidad estacionaria (en adelante vector). Un vector se utiliza para calcular las probabilidades de transición (estado futuro) cuando no se conoce en qué estado se encuentra el sistema. Dichos vectores se obtienen de las matrices de transición (Apéndice 2, Ecuación 4).

Un vector cumple la función de determinar el punto de equilibrio de un modelo de Markov, es decir, permite calcular la probabilidad de cambio del sistema (dos o más pasos adelante en el futuro); además, permite encontrar las probabilidades estacionarias del modelo y determinar en qué es- tado puede encontrarse la cadena cuando lleva un periodo prolongado de funcionamiento.

\section{Problema}

Este estudio se propone modelar la dinámica de transición de un grupo de 34 niños de 4 años de edad frente a una tarea que implica hacer clasificaciones multiplicativas. La pregunta que se formula es la siguiente: ¿Subyacen patrones de variabilidad en los desempeños de los niños?

\section{Método}

\section{Participantes}

Treinta y cuatro niños (19 niñas y 15 niños, $M_{\text {edad }}=4$ años (rango de edad: 4 años y 4 años y 4 meses) fueron seleccionados de seis jardines infantiles de la ciudad de Cali (Colombia). Los jardines infantiles son instituciones educativas privadas pertenecientes a un estrato socioeconómico alto (estratos 5 y 6) de acuerdo con la clasificación del Departamento Administrativo Nacional de Estadística (DANE). Se tomó un número similar de niños y niñas de cada jardín como criterio para no introducir sesgos en la distribución de los participantes en función del género ni del lugar de procedencia. La participación de los niños en el estudio dependió del consentimiento escrito de sus padres y de la aprobación correspondiente de la dirección de la institución educativa.

\section{Materiales}

La tarea LAS CARITAS es una situación de resolución de problemas utilizada en estudios de nuestro equipo (Ossa \& Puche-Navarro, 2010), derivada con mucha libertad y lejanamente de la tarea de las 20 preguntas (Mosher \& Hornsby, 1966). El objetivo del juego es encontrar la carita, en el menor número de movimientos: haciendo click sobre las caritas o formulando preguntas que pueden responder con un sí o con un no. La tarea funciona como un juego de adivinanzas y preguntas, porque los 


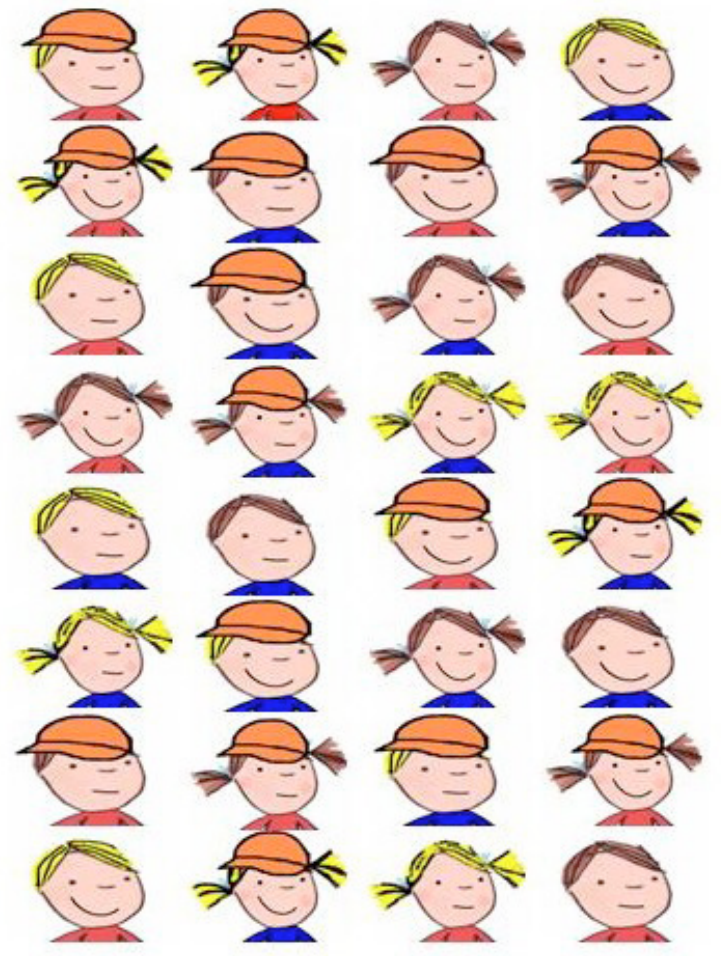

Figura 1. Tarea de clasificación LAS CARITAS.

Fuente: elaboración propia.

niños participantes deben descubrir la carita que el investigador tiene en mente.

Cada pantalla de la tarea está conformada por 32 caritas (Figura 1) que se configuran a partir de la potencia de cinco variables dicotómicas: género (niño o niña), color de pelo (castaño o amarillo), gorro (con gorro o sin gorro), color de camisa (azul o roja) y gesto (sonrisa o no sonrisa). Cada una de ellas con dos valores posibles. Cada uno de los valores de las variables se denomina atributo y se definen como los criterios de clasificación que delimitan el espacio de búsqueda a partir de las preguntas que los niños pueden hacer.

\section{Procedimiento}

La tarea fue presentada a los niños y a las niñas en un formato de juego digital (que en el monitor de un computador adquiere una dimensión aproximada de 40 × $50 \mathrm{~cm}$ ). El procedimiento de cada aplicación es individual y tiene una duración que oscila entre 5 y 15 minutos (aproximadamente). La recolección de los datos se realizó en el jardín infantil, utilizando un computador portátil donde previamente se instaló el software de la tarea. Frente a la pantalla del computador se formularon breves conversatorios, individuales y semi-estandarizados entre niño y experimentador. Durante la fase de familiarización, se verificó que cada participante comprendiera el objetivo de la tarea: se identificaron cada uno de los atributos y se aseguraron aspectos técnicos como el uso del ratón del computador.

\section{Registros y medición}

Los registros de los desempeños se realizaron con el software Rostrox (Ossa \& Puche-Navarro, 2010).

TABLA 1

Estados que caracterizan los diferentes tipos de búsqueda

\begin{tabular}{|c|c|}
\hline Estados & Descriptores \\
\hline \multirow[b]{2}{*}{ Aleatorio (A) } & La búsqueda es aleatoria o se hacen barridos espaciales (criterios no confirmados vía feedback) \\
\hline & $\begin{array}{l}\text { Se sigue } 1 \text { criterio (o más) que no corresponde(n) al personaje (criterios no confirmados vía feedback y siguen una } \\
\text { tendencia sesgada) }\end{array}$ \\
\hline \multirow{2}{*}{$\begin{array}{l}1 \text { criterio de búsqueda } \\
\text { (1C) }\end{array}$} & $\begin{array}{l}\text { Formulación de } 1 \text { pregunta y seguimiento de un } 1 \text { criterio confirmado vía feedback que corresponde al personaje los } 4 \\
\text { criterios restantes son aleatorios }\end{array}$ \\
\hline & $\begin{array}{l}\text { Formulación de } 1 \text { pregunta y seguimiento de un } 1 \text { criterio confirmado vía feedback que corresponde al personaje, } 3 \\
\text { criterios aleatorios y } 1 \text { criterio que no corresponde }\end{array}$ \\
\hline \multirow[b]{2}{*}{$\begin{array}{l}2 \text { o más criterios de } \\
\text { búsqueda }(2 \mathrm{C})\end{array}$} & Formulación de 2 preguntas y seguimiento de un 2 criterios confirmado vía feedback que corresponden al personaje \\
\hline & $\begin{array}{l}\text { Formulación de } 3 \text { o más preguntas y seguimiento de un } 3 \text { o más criterios confirmado vía feedback que corresponden al } \\
\text { personaje }\end{array}$ \\
\hline
\end{tabular}

Nota. Los estados se definen a partir del tipo de búsqueda que realiza el niño. Los tipos de búsqueda se operacionalizan a partir de los descriptores: Indicadores tales como la formulación de preguntas fueron corroborados con la frecuencia de uso de la información a lo largo de la trayectoria de búsqueda del niño.

Fuente: elaboración propia. 
El programa Rostrox genera para cada aplicación una hoja de cálculo Excel donde se registran las elecciones y las preguntas que el niño hace al experimentador.

\section{Diseño}

Se trata de un estudio de mediciones repetidas (series temporales cortas) e interrumpidas (Arnau, 1999). A lo largo de dos meses se hacen cuatro aplicaciones (con intervalos de dos semanas), en cada aplicación se presentan cinco experimentos. Se trata de una estrategia microgenética de recolección de información diseñada con el propósito de lograr mediciones intra-aplicaciones y transversales del desempeño de los niños (Siegler \& Svetina, 2002, p. 196).

\section{Puntajes}

En este estudio, se utiliza una escala de seis niveles que definen tres estados posibles o clases de búsqueda que los niños pueden desplegar. Se trata de una adaptación de la escala de medición que se ha utilizado en el equipo de investigación de PucheNavarro (Ossa, 2011; Ossa \& Puche-Navarro, 2010; Puche-Navarro \& Ossa, 2006).

\section{Resultados}

Los resultados se presentan siguiendo el itinerario de organización de los datos. En primer lugar, se presentan los tres grupos de niños clasificados según su desempeño por la técnica $\mathrm{k}$-medias². A partir de esta clasificación, es decir, los clústeres que definen tres grupos de niños diferenciados por su desempeño (tipos de búsqueda), se obtienen tres matrices de transición y los vectores que resu-

2 Esta técnica permite clasificar los niños en diferentes grupos a partir del tipo de búsqueda que los niños hacen. El número de grupos se ha definido con base en las distancias euclidianas entre cada niño y los centroides definidos por el algoritmo: un centroide es un punto que define el centro geométrico de cada uno de los grupos. men las tendencias de las movilizaciones. En una segunda fase, se presentan las dinámicas de transición frente a cuatro aplicaciones de la tarea lo que permite configurar un patrón de movilizaciones de cada grupo de niños.

Al conjunto de los 34 niños de 4 años de edad se le aplicó la técnica k-medias que arrojó tres grupos de niños con desempeños claramente diferenciados por el tipo de búsqueda. Los tres tipos de búsqueda son: búsqueda aleatoria o por barridos espaciales (en adelante A), búsqueda siguiendo un criterio de clasificación (en adelante 1C) y búsqueda siguiendo dos o más criterios de clasificación (en adelante 2C). Los grupos identificados fueron: 16 niños con tendencia a realizar búsquedas aleatorias o por barridos espaciales, 8 niños que realizan indistintamente las tres clases de búsqueda y 10 niños con tendencia a realizar búsquedas que implican utilizar dos o más criterios de clasificación.

\section{Movilizaciones Grupo 1}

A partir de la clasificación k-medias se identificó que este grupo tiende a realizar búsquedas aleatorias o por barridos espaciales (A). La matriz de transición confirma esta tendencia y arroja un panorama más completo de las movilizaciones (Figura 1). De acuerdo a los procesos markovianos se observa un proceso cuasi-absorbente caracterizado por la

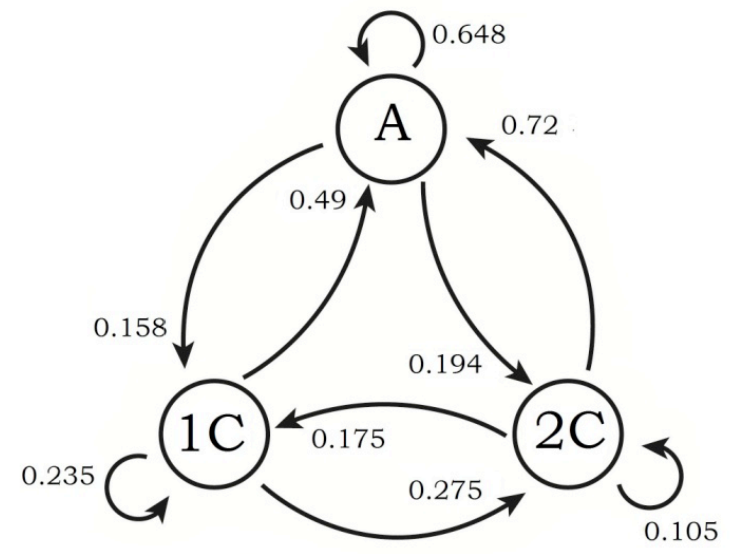

Figura 2. Diagrama de transición de la Matriz 1 (Apéndice 1).

Fuente: elaboración propia. 
tendencia a permanecer y a retornar a un tipo de búsqueda aleatorio (como se ha mencionado anteriormente una cadena de Markov se compone de diferentes estados en los cuales se pueda encontrar el proceso, en el caso del presente estudio los estados se han definido a partir de los tipos de búsqueda realizados por los niños).

En la Figura 2 las movilizaciones entre diferentes tipos de búsqueda se expresan en términos de probabilidades de transición. Estas probabilidades permiten calcular el cambio o la permanencia en un mismo estado frente a diferentes intentos de resolución de la tarea. La lectura de la Figura 2 se hace observando la probabilidad asociada a cada arco dirigido (flecha), por ejemplo: al tomar como punto de referencia la búsqueda aleatoria (A), se observa que una flecha retorna al mismo estado, esto se denomina bucle y significa que la probabilidad de hacer el mismo tipo de búsqueda en el siguiente intento es de $64.8 \%$. También se observa que del estado búsqueda aleatoria (A) parte una flecha dirigida al estado 1 Criterio (1C), lo que significa que la probabilidad asociada a dicho cambio de estado es de $15.8 \%$. Finalmente, del estado búsqueda aleatoria (A) parte una flecha dirigida al estado 2 Criterio (2C) con una probabilidad de transición de $19.4 \%$. De la matriz se obtiene el vector que arroja la siguiente dinámica de cambio: $v=\left(\begin{array}{lll}0.634 & 0.175 & 0.191\end{array}\right)$. La probabilidad de hacer

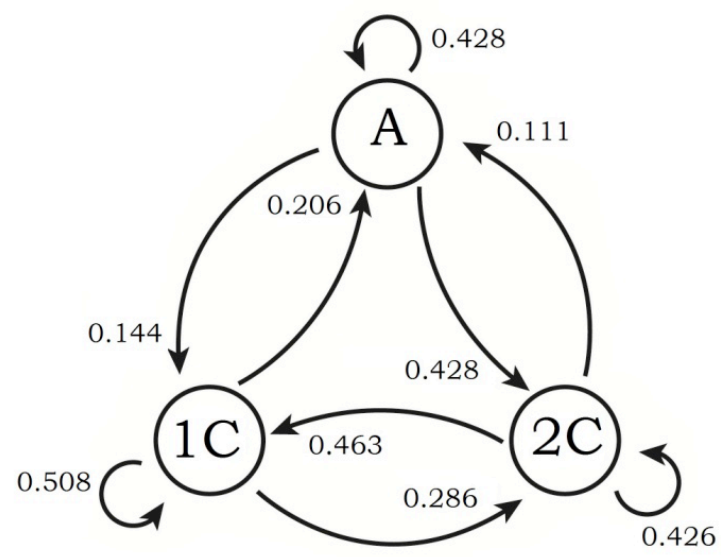

Figura 3. Diagrama de transición de la Matriz 2 (Apéndice 1).

Fuente: elaboración propia. una búsqueda aleatoria es de $63.4 \%$, la probabilidad de hacer una búsqueda utilizando 1 criterio es de $17.5 \%$ y la probabilidad hacer una búsqueda utilizando dos criterios es de $19.1 \%$. Esta probabilidad confirma la tendencia del grupo a permanecer en una búsqueda aleatoria.

\section{Movilizaciones Grupo 2}

Esta dinámica de transición se caracteriza porque tiende a cambiar de tipo de búsqueda frente a cada intento. Es decir, se mueve con casi la misma probabilidad entre las búsquedas (1C) y (2C), en menor grado pasa al estado (A). Desde el punto de vista de la variabilidad esto significa que los cambios son irregulares y con poca probabilidad de estabilizarse en uno de los estados de la cadena de Markov.

En el diagrama de transición (Figura 3) se observa que cuando este grupo realiza una búsqueda $(\mathrm{A})$ la probabilidad de hacer lo mismo en el siguiente intento es de $42.8 \%$, la probabilidad de hacer una búsqueda (1C) es de $14.4 \%$ y la probabilidad de hacer una búsqueda (2C) de $42.8 \%$. De la matriz se obtiene el vector que arroja la siguiente dinámica de cambio: $v=\left(\begin{array}{lll}0.22 & 0.411 & 0.369\end{array}\right)$. La probabilidad de que este grupo haga una búsqueda (A) es de $22 \%$, la probabilidad de hacer una búsqueda (1C) es de $41.1 \%$ y la probabilidad hacer una búsqueda (2C) es de $36.9 \%$.

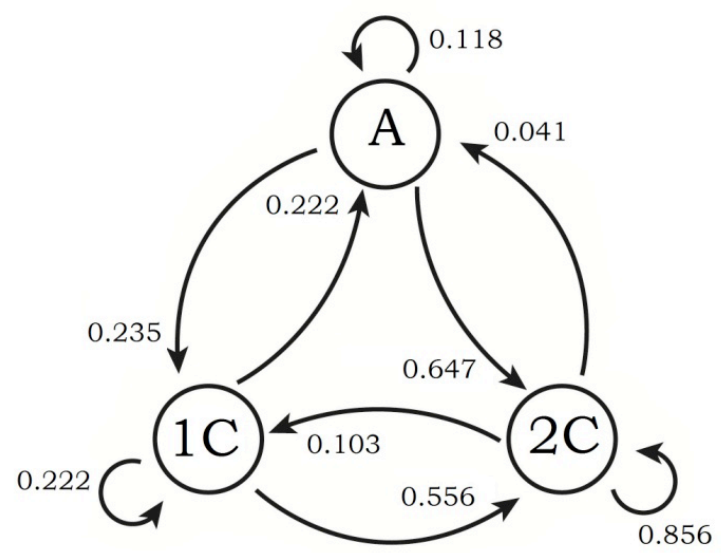

Figura 4. Diagrama de transición de la Matriz 3 (Apéndice 1).

Fuente: elaboración propia. 


\section{Movilizaciones Grupo 3}

Esta dinámica de transición se caracteriza porque muestra una marcada tendencia a realizar búsquedas, utilizando dos criterios o más (2C). La cadena de Markov tiende a un proceso de absorbencia por la recurrencia a este tipo de búsqueda.

En el diagrama de transición (Figura 4), se observa que cuando el grupo realiza una búsqueda (2C) la probabilidad de hacer el mismo tipo de búsqueda en el siguiente intento es de $85.6 \%$, la probabilidad de hacer una búsqueda (1C) es de $10.3 \%$ y la probabilidad de pasar a una búsqueda (A) es de $4.1 \%$. De la matriz se obtiene el vector que arroja la siguiente dinámica de cambio: $v=\left(\begin{array}{lll}0.07 & 0.127 & 0.803\end{array}\right)$. La probabilidad de que este grupo haga una búsqueda (A) es de $7 \%$, la probabilidad de hacer una búsqueda (1C) es de $12.7 \%$ y la probabilidad hacer una búsqueda (2C) es de $80.3 \%$.

\section{Dinámicas de transición: origen de los patrones de variabilidad}

Con el propósito de observar la dinámica de transición de los grupos, se hace un análisis de variabilidad entre aplicaciones. Debe recordarse que fueron cuatro aplicaciones en cuatro momentos diferentes (cada 2 semanas). En cada aplicación se presentó la tarea cinco veces, con intervalos de tiempo regulados por el niño. En cada intento se cambió la distribución espacial de los objetos y, en este sentido, la tarea fue privilegiada porque siempre le presentó al niño el mismo problema pero con diferentes formas. Es así como para cada aplicación se obtiene una matriz de transición y un vector.

De cada grupo se obtienen cuatro vectores que capturan la dinámica de las movilizaciones. En la Figura 5 se presentan esas dinámicas internas a lo largo de cuatro aplicaciones. Este proceso se hace siguiendo las probabilidades de transición en tres dimensiones del cambio, es decir, los tres tipos de búsqueda o estados del modelo: Aleatorio (A), 1 criterio (1C) y 2 criterios (2C) de clasificación.

Se percibe que la dinámica de variabilidad restringida refleja transiciones estables en el tiempo y probabilidades estacionarias con fluctuaciones suavizadas en las probabilidades estacionarias de los vectores. La probabilidad de hacer una búsqueda (A) en la 1. a aplicación es $64.7 \%$, en la 2. a aplicación es $59.5 \%$, en la $3 .^{\text {a }}$ aplicación es $65 \%$ y en la 4. a aplicación es $55.4 \%$. La probabilidad de hacer una búsqueda (1C) en la 1. a aplicación es $11.3 \%$, en la 2. ${ }^{\text {a }}$ aplicación es $27 \%$, en la 3. a aplicación es $16 \%$ y en la $4 .^{\text {a }}$ aplicación es $25 \%$. Finalmente, la probabilidad de hacer una búsqueda (2C) en la $1 .^{2}$ aplicación es $24 \%$, en la 2. a aplicación es $27 \%$, en la 3. a aplicación es $16 \%$ y en la 4. a aplicación es $19.6 \%$.

La dinámica de variabilidad fluctuante refleja transiciones irregulares en el tiempo y probabili-

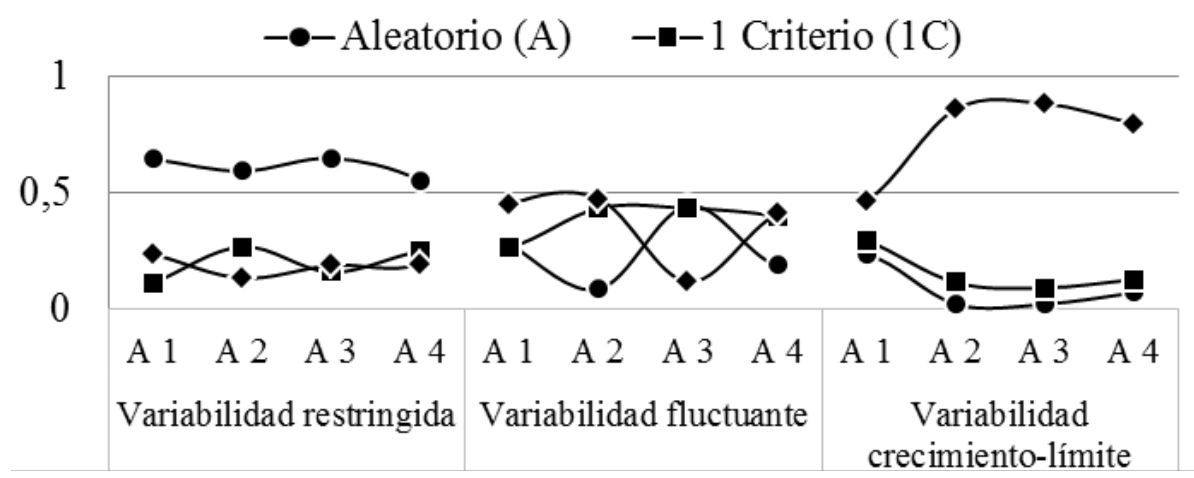

Figura 5. Dinámicas de transición de cada uno de los patrones de variabilidad.

Nota. Se observan tres patrones de variabilidad (restringida, fluctuante y de crecimiento límite) frente a cuatro aplicaciones de la tarea.

Fuente: elaboración propia. 
dades estacionarias con cambios oscilantes en las probabilidades de transición de los vectores. La búsqueda (A) en la 1. a aplicación es $28.2 \%$, en la 2. a aplicación baja a $8.8 \%$, en la 3. a aplicación sube a $44.7 \%$ y en la $4 .{ }^{\mathrm{a}}$ aplicación baja a $19 \%$. La probabilidad de hacer una búsqueda (1C) en la 1. a aplicación es $26.6 \%$, en la 2. ${ }^{\mathrm{a}}$ aplicación sube $43.6 \%$, en las 3. ${ }^{\mathrm{a}}$ y $4 .^{\mathrm{a}}$ aplicaciones permanece en $43.5 \%$ y $40 \%$, respectivamente. La probabilidad de hacer una búsqueda (2C) en la $1 .^{\text {a }}$ aplicación es $45 \%$, en la $2^{a}$ aplicación permanece en $47.6 \%$, en la 3. a aplicación baja a 11.8 \% y en el 4. a aplicación sube a $41 \%$.

La dinámica de variabilidad crecimiento-límite refleja un cambio en las probabilidades de transición de los vectores. A partir de la 2. a aplicación se observa una marcada tendencia a realizar búsquedas (2C) y se reducen ostensiblemente las probabilidades de hacer búsquedas (A) y (1C). Se debe resaltar que el punto de origen es muy semejante las probabilidades de transición observada en la dinámica fluctuante, antes mencionada, pero la trayectoria diverge ostenciblemente: la búsqueda (A) en la 1. a aplicación es $23.6 \%$, en la 2. ${ }^{a}$ aplicación baja a $2.3 \%$, en la 3. a aplicación permanece en $2.3 \%$ y en la $4 .{ }^{a}$ aplicación sube ligeramente a $7.2 \%$. La probabilidad de hacer una búsqueda (1C) en la $1 .^{\mathrm{a}}$ aplicación es $29.6 \%$, en la 2. a aplicación baja $11.16 \%$, en la 3. ${ }^{\text {a }}$ baja nuevamente a $9.3 \%$ y en la 4. ${ }^{\mathrm{a}}$ aplicación sube a $12.9 \%$. La probabilidad de hacer una búsqueda (2C) en la 1 . $^{\text {a aplicación es }}$ $46.8 \%$, en la $2 .^{\text {a }}$ aplicación sube abruptamente a $86.1 \%$, en la 3. a aplicación permanece en $88.4 \%$ y en la 4. a aplicación declina a $79.9 \%$.

\section{Discusión}

A partir de las diferentes trayectorias que toma el sistema, y como se ha puesto en evidencia en la Figura 5, se puede describir la variabilidad como el resultado de un proceso de desequilibrio cognitivo, al pasar de un punto de equilibrio a otro.

La variabilidad es la evidencia de un proceso de desequilibrio cognitivo y se puede observar a partir de las diferentes trayectorias que toma el sistema para pasar de un punto de equilibrio a otro. Puede afirmarse que la variabilidad es la estela de una trayectoria observada por fuera de cualquier punto de equilibrio. Desde este punto de vista, se debe preguntar: ¿Qué información arrojan las matrices de transición y los vectores de probabilidad estacionaria en relación con la variabilidad?

El aporte de este tratamiento es identificar tres tipos de regularidades o patrones de variabilidad (a partir de las matrices de transición y los vectores de probabilidad estacionaria). Por ejemplo, la dinámica de variabilidad restringida refleja la tendencia a permanecer en un punto de equilibrio: a partir del vector que resume la dinámica de transición de este tipo de variabilidad, se identificó que la probabilidad de salir de este punto de equilibrio es del $36.6 \%$ y que la probabilidad de retornar o de permanecer en este estado de equilibrio es del $63.4 \%$.

En la dinámica de variabilidad fluctuante, se observa que la probabilidad de permanecer o de retornar al punto de equilibrio inicial es de $22 \%$. Con esta dinámica se empiezan a esbozar otros puntos de equilibrio. Estos nuevos puntos de equilibrio son inestables ya que se observa que la probabilidad de permanecer en ellos está alrededor del $50 \%$ (como se evidencia en la matriz de transición de dicho grupo). En el vector se evidencia la tendencia a movilizarse entre los nuevos estados de equilibrio es del $78 \%$.

En la dinámica de transición denominada como crecimiento-límite se observa que la probabilidad de pasar a un nuevo estado de equilibrio es de $80.3 \%$ y la probabilidad de permanecer en dicho punto de equilibrio es de $85.6 \%$ (Figura 4). El retorno a un punto de equilibrio diferente es de $19.7 \%$. El campo de equilibrio, en esta dinámica de transición, se define a partir de las búsquedas que utilizan dos o más criterios de clasificación.

Ahora bien, si se afirma que la variabilidad es la evidencia de un desequilibrio cognitivo, la pregunta que subyace es: ¿Cuál es la relación entre la variabilidad y las diferentes formas de equilibrio? Como se ha observado, se encuentra una dinámica de transición anclada a la búsqueda aleatoria donde las salidas a otro estado implican un retorno necesario al estado de equilibrio anterior; y dos dinámicas de transición que pese a tener puntos de inicio casi 
similares la primera de ellas, la fluctuante, pasa por todos los estados de manera regular mientras la segunda, la de cambio, pasa por dichos estados de manera transitoria para anclarse en un nuevo estado de equilibrio.

La tarea de clasificación LAS CARITAS implica, para su solución "ideal", la coordinación multiplicativa de diferentes fuentes de información. Como se ha mencionado, un punto de equilibrio se evidencia cuando los niños hacen búsquedas aleatorias. Una búsqueda aleatoria se regula por la disposición espacial de los objetos mas no por sus dimensiones cualitativas.

Para los grupos de niños fluctuantes, la naturaleza del problema y la necesidad de hacer coordinaciones multiplicativas perturba el punto de equilibrio (búsqueda aleatoria). La fuente de variación está dada en el proceso que implica desacoplar el sistema total en sus principales componentes y a su turno hacer una integración de las dimensiones y proyectarlas a un objeto. El primer proceso tiene un referente concreto, pero el segundo opera en el nivel de la abstracción y no hay un punto de apoyo en lo real. La coordinación de dichos procesos refleja la naturaleza de la variabilidad de este grupo fluctuante.

\section{Referencias}

Arnau, J. (1999). Series temporales cortas y mínimos cuadrados generalizados: análisis de la intervención. Revista de Metodología de las Ciencias del Comportamiento, 1(2), 119-135.

Combariza, E. \& Puche-Navarro, R. (2009). El uso de la wavelet para el estudio de los funcionamientos inferenciales en niños pequeños. En R. Puche-Navarro (Comp.), iEs la mente no lineal? (pp. 111-133). Cali: Programa Editorial Universidad del Valle.

Courage, M. \& Howe, M. (2002). From infant to child: The dynamics of cognitive change in the second year of life. Psychological Bulletin, 128, 250-277.

Goswami, U. (2001). Cognitive development: No stages please-we're British. British Journal of Psychology, 92(1), 257-277.

Kerlinger, F. \& Lee, H. (2002). Fundamentos de medición. En F. Kerlinger \& H. Lee (Eds.), Inves- tigación del comportamiento (4a. ed., pp. 565-580). México: McGraw-Hill.

Miller, P. H. (2002). Order in variability, variability in order. Why it matters for theories of development. Human Development, 45(3), 161-166.

Piaget, J. (1979). El mecanismo del desarrollo mental. Madrid: Editora Nacional.

Piaget, J. (2000). La equilibración de las estructuras cognitivas. México: Siglo XXI.

Pinker, S. (2001). Buenas ideas. En S. Pinker (Ed.), ¿Cómo funciona la mente? (pp. 387-463). Bogotá: Planeta.

Mosher, F. A. \& Hornsby, J. R. (1966). On asking questions. In J. S. Bruner et al. (Eds.), Studies in cognitive growth (pp. 86-102). New York: Wiley.

Ossa, J. C. (2011). Funcionamiento cognitivo: Un inextricable juego de pérdidas y ganancias. Acta Colombiana de Psicología, 14(2), 45-55.

Ossa, J. C. \& Puche-Navarro, R. (2010). Modelos bayesianos y funcionamientos inferenciales complejos. Acta Colombiana de Psicología, 13(2), 119-128.

Puche-Navarro, R. (2003). Procesos de desarrollo, cambio y variabilidad. En R. Puche-Navarro (Ed.), El niño que piensa y vuelve a pensar (pp. 17-50). Cali: Artes Gráficas del Valle.

Puche-Navarro, R. (2008). Érase una vez el desarrollo. En J. Larreamendy-Joerns, R. Puche-Navarro \& A. Restrepo-Ibiza (Comps.), Claves para pensar el cambio: Ensayos sobre psicología del desarrollo (pp. 29 69). Bogotá: Editorial Universidad de los Andes.

Puche-Navarro, R. \& Ossa, J. C. (2006). ¿Qué hay de nuevo en el método microgenético? Más allá de las estrategias y más acá del funcionamiento cognitivo del sujeto. Suma Psicológica, 13(2), 117-137.

Puche-Navarro, R., Ossa J. C. \& Guevara, M. (2006). La resolución infantil de problemas, iuna alternativa integradora? Educación y Pedagogía, 18(46), 167-189.

Rose, L. T. \& Fischer, K. W. (2009). Dynamic systems theory. En R. A. Shweder (Ed.), The child: An encyclopedia companion (pp. 264-265). Chicago: University of Chicago Press.

Siegler, R. S. (1994). Cognitive variability: A key to understanding cognitive development. Current Directions in Psychological Science, 3(1), 1-5. 
Siegler, R. S. (1995). How does change occur: A microgenetic study of number conservation. Cognitive Psychology, 28, 225-273.

Siegler, R. S. (2002a). Microgenetic studies of selfexplanations. En N. Granott \& J. Parziale (Eds.), Microdevelopment: Transition processes in development and learning (pp. 31-58). New York: Cambridge University.

Siegler, R. S. (2002b). Variability and infant development. Infant Behavior E Development, 25(4), 550-557.

Siegler, R. S. \& Svetina, M. (2002). A microgenetic/ cross-sectional study of matrix completion: Comparing short-term and long-term change. Child Development, 73(3), 793-809.

Siegler, R. S. \& Svetina, M. (2006). What leads children to adopt new strategies? A microgenetic/cross sectional study of class inclusion. Child Development, 77(4), 997-1015. van Dijk, M. \& van Geert, P. (2007). Wobbles, humps and sudden jumps: A case study of continuity, discontinuity and variability in early language development. Infant and Child Development, 16(1), 7-33. van Geert, P. \& van Dijk, M. (2002). Focus on variability: New tools to study intra-individual variability in developmental data. Infant Behavior and Development, 25(4), 340-374.

Visser, I., Raijmakers, M. E. J. \& Molenaar, P. C. M. (2002). Fitting hidden Markov models to psychological data. Scientific Programming, 10(3), 185-199.

Yan, Z. \& Fischer, K. (2002). Always under construction: Dynamic variations in adult cognitive microdevelopment. Human Development, 45(3), 141-160.

Yan, Z. \& Fischer, K. (2007). Pattern emergence and pattern transition in microdevelopmental variation: Evidence of complex dynamics of developmental processes. Journal of Developmental Processes, 2(2), 39-62. 


\section{Apéndice 1}

Matrices de Transición de cada uno de los grupos

\begin{tabular}{|c|c|c|c|c|c|c|c|c|c|c|c|}
\hline \multicolumn{4}{|c|}{ Matriz 1} & \multicolumn{4}{|c|}{ Matriz 2} & \multicolumn{4}{|c|}{ Matriz 3} \\
\hline \multirow{3}{*}{$P=$} & 0.648 & 0.158 & $0.194)$ & \multirow{3}{*}{$P=$} & 0.428 & 0.144 & $0.428)$ & \multirow{3}{*}{$P=$} & 0.118 & 0.235 & $0.647)$ \\
\hline & 0.49 & 0.235 & 0.275 & & 0.206 & 0.508 & 0.286 & & 0.222 & 0.222 & 0.556 \\
\hline & 0.72 & 0.175 & 0.105 & & 0.111 & 0.463 & 0.426 & & 0.041 & 0.103 & $0.856)$ \\
\hline
\end{tabular}

\section{Apéndice 2}

\section{Ecuaciones del modelo de Markov (Chapman-Kolmogorov)}

Ecuación 1: $P\left(X_{n+1}=j / X_{n}=i\right)=p(i, j)$

Ecuación 2: $P\left(X_{n+1}=j / X_{n}=i, X_{n-1}=i_{n-1}, \ldots, X_{0}=i_{0}\right)=P\left(X_{n+1}=j / X_{n}=i\right)$

Ecuación 3: $P\left(X_{m}=j / X_{0}=i\right)=p^{m}(i, j)$

Ecuación 4: $x \vec{q}=\left(P\left(x_{t}=e_{1}\right), P\left(x_{t}=e_{2}\right), \ldots P\left(x_{t}=e_{n}\right)\right)$ 